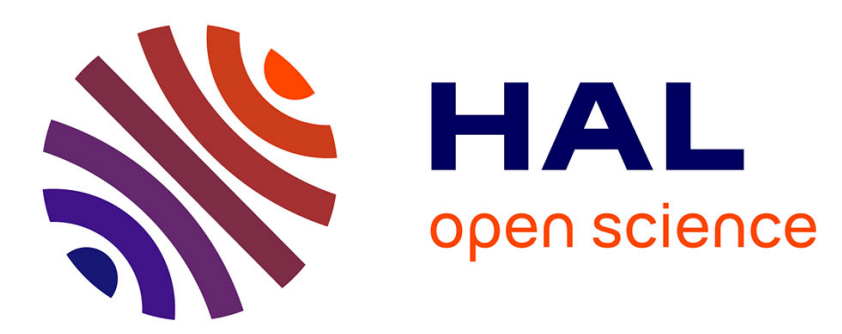

\title{
Multistable interaction between a spherical Brownian particle and an air-water interface
}

Stefano Villa, Antonio Stocco, Christophe Blanc, Maurizio Nobili

\section{To cite this version:}

Stefano Villa, Antonio Stocco, Christophe Blanc, Maurizio Nobili. Multistable interaction between a spherical Brownian particle and an air-water interface. Soft Matter, 2020, 16, pp.960. 10.1039/C9SM01472A . hal-02460100

\section{HAL Id: hal-02460100 https://hal.science/hal-02460100}

Submitted on 23 Sep 2020

HAL is a multi-disciplinary open access archive for the deposit and dissemination of scientific research documents, whether they are published or not. The documents may come from teaching and research institutions in France or abroad, or from public or private research centers.
L'archive ouverte pluridisciplinaire HAL, est destinée au dépôt et à la diffusion de documents scientifiques de niveau recherche, publiés ou non, émanant des établissements d'enseignement et de recherche français ou étrangers, des laboratoires publics ou privés. 


\title{
Journal Name
}

\section{ARTICLE TYPE}

Cite this: DOI: $00.0000 / x x x x x x x x x x$

\section{Multistable Interaction between a Spherical Brownian Particle and an Air-Water Interface}

\author{
Stefano Villa, ${ }^{a}$ Antonio Stocco, ${ }^{b}$ Christophe Blanc, ${ }^{a}$ and Maurizio Nobili ${ }^{* a}$
}

Received Date

Accepted Date

DOI: $00.0000 / x x x x x x x x x x$

\begin{abstract}
We report the measurement of the interaction energy between a charged Brownian polystyrene particle and an air-water interface. The interaction potential is obtained from Boltzmann equation by tracking particle interface distance with a specifically designed Dual-Wave Reflection Interference Microscopy (DW-RIM) setup. The particle has two equilibrium positions located at few hundreds of nanometers from the interface. The farthest position is well accounted by a DLVO model complemented by gravity. The closest one, not predicted by current models, more frequently appears in water solutions at relatively high ions concentrations, when electrostatic interaction is screened out. It is accompanied by a frozen rotational diffusion dynamics that suggests an interacting potential dependent on particle orientation and stresses the decisive role played by particle surface heterogeneities. Building up on both such experimental results, the important role of air nanobubbles pinned on the particle interface is discussed.
\end{abstract}

\section{Introduction}

The interactions between a liquid interface and solid particles play a central role in colloidal science and in a large number of applications in the field. They for example determine the stability of emulsions largely employed in food and cosmetic products. 1 Moreover, they also have a crucial importance in flotation techniques in mineral and water remediation processes. In flotation, air bubbles are driven in water columns charged with solid waste or mineral particles in order to remove them from the water via bubble interface adsorption. 2

Despite the large field of applications, direct measurements of interaction between particles and air-liquid interfaces remain relatively scarce and difficult to perform. Research in the field has been restricted to flat solid surfaces interacting with air-bubbles via a thin film of liquid. In such systems, experimental results obtained with Surface Force Apparatus agree with DLVO models considering repulsive van der Waals and double-layers interactions. $\frac{34}{4}$ The latter arises from the negative potential of the airwater interface, the origin of which is still debated but is probably due to an excess of $\mathrm{OH}^{-}$ions, $\frac{3 \sqrt{56}}{6}$ and the negative electric charges present on the employed solid surfaces. These studies have also been extended towards geometries more similar to the ones of the applications to flotation as the ones where solid particles attached to an AFM tip are forced to move in the vicinity of an air bubble. 7

\footnotetext{
${ }^{a}$ Laboratoire Charles Coulomb (L2C), University of Montpellier, CNRS, Montpellier, France.E-mail: maurizio.nobili@umontpellier.fr

${ }^{b}$ Institut Charles Sadron (ICS), University of Strasbourg, CNRS, Strasbourg, France.
}

The interaction between a liquid interface and a freely diffusing colloid has been only recently addressed in the case of a polystyrene particle close to an oil-water interface using Total Internal Reflection Microscopy (TIRM). 8 The particle-interface interaction has been extracted from the distribution of distances using Boltzmann equation. It shows a single minimum located at hundreds of nanometers from the interface resulting from the combination of gravitational, attractive van der Waals and repulsive electric double-layers interactions.

In this work, we focus on the interaction between spherical micro-particles freely diffusing close to an air-water interface and the interface itself. This interaction is extracted from the threedimensional (3D) tracking of the Brownian motion of particles in the proximity of the interface. In our case TIRM technique is not suitable due to the positive mismatch of the refractive index going from air to water. ${ }^{[8] 9}$ The particle interface distance and eventually its orientation with respect to the interface are measured using a Dual-Wave Reflection Interference Microscopy (DW-RIM) setup 10 optimized for liquid interfaces. Differently from holographic methods, 13 such technique allows to track the particle-interface distance with a precision of the order of $10 \mathrm{~nm}$ and without requiring any calibration or assumption on the interface location.

\section{Experimental}

\subsection{Sample preparation}

The experimental cell consists in an hollow glass cylinder with inner radius $a_{c}=4 \mathrm{~mm}$ glued on a glass slide on the top and exposed to air on the bottom. It is filled with a water solution 
whose volume is fixed in order to completely fill the cylinder and obtain a planar air-liquid interface. The contact line between the solution, the glass and the air is pinned on the lower inner edge of the cylinder. The water solution is therefore the upper phase while air is the lower one. This configuration allows the particles suspended in the solution to sediment towards the air-liquid interface. In order to reduce water evaporation the cell and the objective are surrounded by an isolated chamber as shown in figure 1

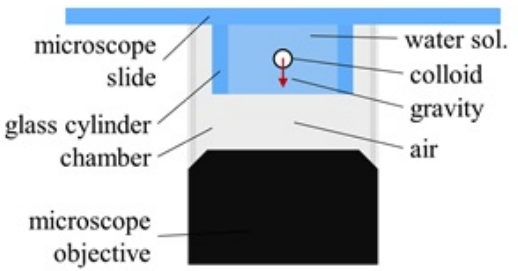

Fig. 1 Sketch of the experimental cell and its environment. The cell is made of an hollow glass cylinder (height and inner radius of $4 \mathrm{~mm}$ ) glued on a microscope slide. Microparticles contained in the water solution sediment because of gravity towards the air-water interface. The cell and the objective are enclosed in a flexible chamber in order to reduce water evaporation and particle drift at the air-liquid interface.

Microparticles are suspended in water solutions at concentrations low enough (less than $10^{-2} \mathrm{~g} / \mathrm{L}$ ) to obtain average distances between particles larger than $100 \mu \mathrm{m}$ when close to the air-liquid interface. Two kinds of polystyrene latex beads (density $\rho_{p s}=1055 \mathrm{Kg} / \mathrm{m}^{3}$ ) with different surface functionalization have been employed. One set has a diameter $2 a=8.7 \pm 0.9 \mu \mathrm{m}$ and is functionalized with sulfate groups (Molecular Probes), while the second one has a diameter $2 a=8.9 \pm 0.1 \mu \mathrm{m}$ and is functionalized with carboxyle groups (Microparticles $\mathrm{GmbH}$ ). AFM measurements of the particle topography revealed that sulfate microparticles present a small number (tens per particle) of craters on their surface with lateral sizes of hundreds of nanometers and a depth of 30-80 nm (figure 2). Since these defects are large enough to be optically detectable, they are used to access to particle orientation, as reported in the Results and discussion section.

In order to know the particles surface charge $\sigma_{q}$, we performed Zeta potential $(\zeta)$ measurements for both sulfate and carboxylated particles (Malvern Instruments, Zetasizer Nano ZS). For the determination of the effective charge associated to a given potential $\zeta$ we used the O'Brien-White-Ohshima method, which is valid under our conditions of monovalent ions and low Zeta potentials $(\zeta \leq 100 \mathrm{mV}) . \sqrt{14}$ The surface charge densities obtained for sulfate and carboxylated particles are respectively $\sigma_{q}=-5 \pm 1 \mathrm{mC} / \mathrm{m}^{2}$ and $\sigma_{q}=-1.1 \pm 0.3 \mathrm{mC} / \mathrm{m}^{2}$.

Since both the air-liquid interface and the particle surface are electrically charged, the particle-interface interaction is expected to depend on the concentration of ions present in the liquid. For this reason, water solutions at different molar concentrations $c$ of dissolved monovalent ions have been investigated, with $c$ ranging from $10^{-6} \mathrm{M}$ up to $10^{-3} \mathrm{M}$. They are prepared as mixtures of deionized water, obtained from a Millipore Milli-Q filtration

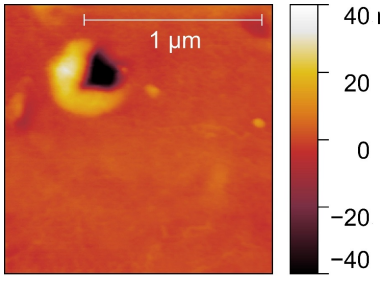

(a)

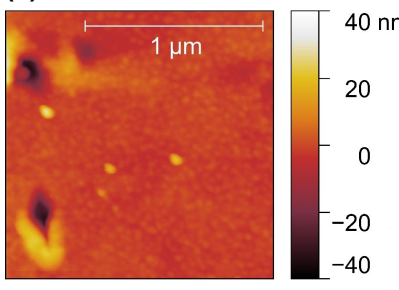

(c)

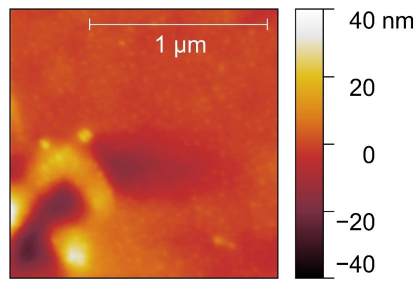

(b)

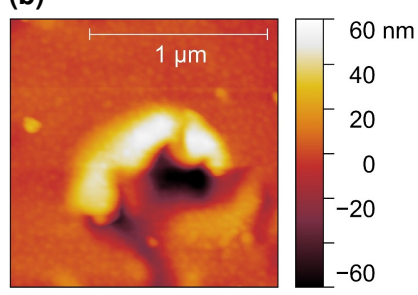

(d)
Fig. 2 (a, b, c, d) Atomic Force Microscopy images of the surface of sulfate particles once the spherical curvature of the particle has been digitally removed. In each image craters can be seen with lateral sizes of hundreds of nanometers and depths of tens of nanometers.

system (nominal resistivity of $18 \mathrm{M} \Omega \cdot \mathrm{cm}$ and density $\rho_{w}=0.997$ $\mathrm{g} / \mathrm{cm}^{3}$ at $25^{\circ} \mathrm{C}$ ), and sodium chloride (Sigma-Aldrich $\mathrm{NaCl}$ anhydrous, $\geq 99 \%$ ). Solution's properties may be in principle influenced during experiments by the adsorption of other ions and contaminants from atmosphere onto the air-water interface. In order to verify the relevance of such effect during experimental time scales, we measured the solutions surface tension at different times through a Profile Analysis Tensiometer (Sinterface, Profile Analysis Tensiometer), as the adsorption of pollutants at the interface is expected to lower its surface tension. ${ }^{15}$ The test has shown that the surface tension remains constant for all the prepared solutions at values between 72 and $72.5 \mathrm{mN} / \mathrm{m}$, in agreement with the expected values for uncontaminated interfaces, 16 during all the time interval of the measurement ( $>4$ hours, more than the duration of one experiment).

\subsection{DW-RIM experimental setup}

The DW-RIM setup is assembled similarly to an inverted microscope working in reflection. A simple sketch of the interference scheme is given in figure 3. a source light beam hits the sample from below and is back-reflected both at the air-liquid interface and at the particle surface. The superposition of the two reflected beams gives rise to an interference pattern from which the tracking of the three translational degrees of freedom of the particles can be made. A two wavelengths illumination is required in order to determine the particle-interface gap distance $d$ from the fringe system. A red (nominal wavelength $\lambda_{R}=625 \mathrm{~nm}$ ) and a blue (nominal wavelength $\lambda_{B}=505 \mathrm{~nm}$ ) monochromatic lightemitting diodes are therefore simultaneously employed as light sources. Their relatively low coherence length of few micrometers allows to obtain the particle-interface interference pattern when the particle is within few micrometers from the interface while it prevents spurious interferences from all other interfaces of the system. An RGB camera is used to record the two wavelengths' 


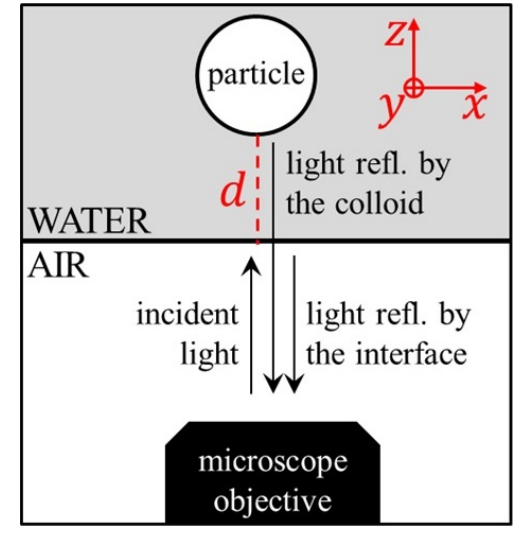

Fig. 3 Reflection geometry for a colloidal particle immersed in a water solution at a gap distance $d$ from the interface with air. The Cartesian reference system is oriented with the $z$ axis along the normal to the interface and the $x y$ plane parallel to the interface. The incident light beam is reflected from both the interface and the particle. The superposition of the reflected beams, collected by the objective, generates the interference pattern.

signals at 35 frames per second. The spectral responses of the red and blue channels of the camera sensor are sufficiently separated to avoid the superimposition of the two colors on the same channel, thus allowing the simultaneous and separate recording of the red and blue fringe systems.

A halogen white light is also employed for the transmission bright field imaging of the particle. It is used to center the particle in the field of view of the camera and to directly measure its radius $a$. This illumination is turned off during the recording of the interference patterns.

\subsection{Particle tracking}

The frame sequence of figure 4 illustrates the time evolution of the interference pattern for a particle moving close to the airliquid interface. As it can be seen, both the center $\left(x_{c}, y_{c}\right)$ and the phase of the pattern change in time, demanding a dedicated image analysis program to track the 3D particle motion. Since the Rayleigh range (about half a micron) is larger than the typical particle-interface distances, the data analysis can be done considering an imaging configuration where both the interface and the particle are simultaneously in focus.

The basic description of RIM image formation consists in considering only incident and reflected beams parallel to the optical axis (see fig 3). The optical path difference between the two reflected beams is therefore $2 n z_{s}(r)$, where $n$ is the refractive index of the water solution and $z_{s}(r)=d+a-a \sqrt{1-r^{2} / a^{2}}$ is the distance along the optical axis between a point of the particle surface at the radial coordinate $r=\sqrt{\left(x-x_{c}\right)^{2}-\left(y-y_{c}\right)^{2}}$ and the air-liquid interface. After simple calculations, the intensity of the interference pattern for a spherical particle results in:

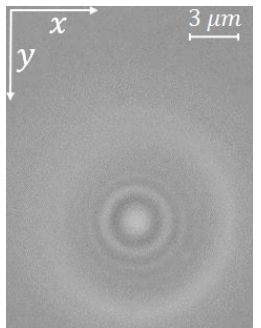

(a)

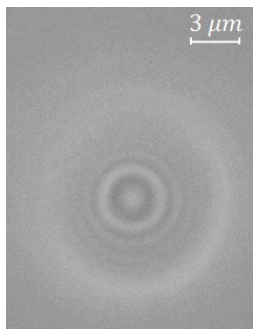

(c)

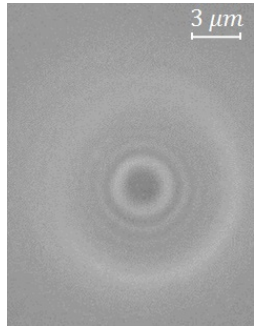

(b)

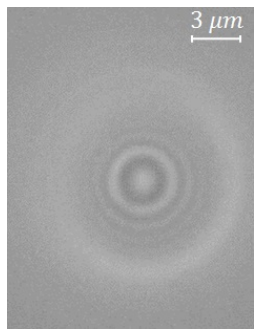

(d)
Fig. 4 (a, b, c, d) Frame sequence of interference pattern of a particle of radius $4.5 \mu \mathrm{m}$ moving close to an air-liquid interface (red channel). The time step between the reported frames is of the order of several seconds.

$$
I(r)=I_{1}+I_{2}+\sqrt{I_{1} I_{2}} \cos \left[\frac{4 \pi n}{\lambda}\left(d+a-a \sqrt{1-\frac{r^{2}}{a^{2}}}\right)\right],
$$

where $I_{1}$ and $I_{2}$ are the intensities of the beams reflected on the interface and on the particle surface respectively.

The basic model predicts an uniform intensity contrast of the fringes, which is obviously not observed in Fig. 4. To detect accurately the center of the pattern, a simple empirical Gaussianmodulation of the sinusoidal part with $e^{-\frac{r^{2}}{2 \sigma^{2}}}$ nevertheless proved to be effective. The fit of the intensity for each $2 \mathrm{D}$ image converges quite rapidly and the center $\left(x_{c}, y_{c}\right)$ is recovered with a typical value sigma kept at $0.6 \mu \mathrm{m}$. With this empirical approach, the position $\left(x_{c}, y_{c}\right)$ of the particle is determined with a sufficient accuracy $(50 \mathrm{~nm})$ but the vertical position is poorly obtained. The next step consists in performing an azimuthal average of the intensity centered in $\left(x_{c}, y_{c}\right)$ in order to obtain the radial profile of the interference intensity $I(r)$ with a higher accuracy.

At this stage, a refined analysis of the intensity pattern should consider the exact propagation of the optical rays as well as other phenomena like the finite spectral width of the LEDs, the numerical aperture and the point spread function of the objective and the transmission/reflection indexes of the involved interfaces as a function of the incident angle. The exact computation of all these effects on the interference pattern for each single frame is a formidable task. 12

Numerical computations taking into account the two main relevant contributions (the presence of tilted rays due to a finite numerical aperture and the curvature of the sphere) satisfactorily account for the intensity distribution ${ }^{[1] 12}$ (see SI) but at the cost 
of lengthy dedicated numerical treatments. On the other side, when obtaining $d$ from the most visible fringe and equation 1 , only a systematic discrepancy from the numerical computation of about $15 \mathrm{~nm}$ is achieved in the particle-interface distance» Accordingly, we considered the radial coordinate of an extremum $r_{e}$ of the intensity. The distance $d$ as a function of $r_{e}$ can be easily obtained from eq. 1 .

$$
d=-a\left(1-\sqrt{1-\left(\frac{r_{e}}{a}\right)^{2}}\right)+\left(m+\frac{j}{2}\right) \frac{\lambda}{2 n}
$$

where $j=0,1$ for interference maxima and minima respectively and $m$ is a natural number representing the number of halfwavelengths contained in the distance between the particle and the interface. From equation 2 $d$ can thus be known up to the additive constant $m \frac{\lambda}{2 n}$ directly from the measurement of the radial coordinate of an extremum The extremum is chosen within a range of $0.5 \mu \mathrm{m}<r<1.5 \mu \mathrm{m}$ in order to minimize the discrepancy between the correct above-mentioned model and the approximated employed one (see Supplementary Information for details).

For a given particle, the value of $m$ is found by comparing the distances $d$ obtained with the two employed wavelengths. The tracking is made both on the red and blue channel of each frame and the time evolution of the distance $d(t)$ is reconstructed for different test values of $m_{R}$ (for the red) and $m_{B}$ (for the blue). The chosen couple of values $m_{R}$ and $m_{B}$ corresponds to the one which minimizes the absolute value of the average over time of the reconstructed distances difference $\left|\left\langle d_{R}-d_{B}\right\rangle_{t}\right|$.

\section{Results and discussion}

\subsection{Measured potential wells}

In figure 5, the distance $d(t)$ of a typical trajectory reconstructed with the described procedure is shown. The particle dynamics exhibits an initial sedimentation regime during which the particle approaches the interface under the influence of gravity. The particle reaches then an equilibrium distance $d_{0}$ at hundreds of nanometers from the air-liquid interface once DLVO repulsion equilibrates gravity. This equilibrium regime eventually ends with the adsorption of the particle at the interface.

Here we focus our attention on the measurement of the particle-interface interaction in the equilibrium regime, when the particle thermally explores the potential around the equilibrium position. A typical result obtained for the particle-interface distance $d$ versus time is reported in figure 6a, where the particle fluctuates in the vertical direction by tens of nanometers around an equilibrium distance located at $d_{0}=410 \mathrm{~nm}$.

From the measured distribution of distances $d$, the potential

\footnotetext{
* Such systematic error is comparable with the experimental error resulting from the other sources of incertitude, detailed in the SI, and can be easily taken into account by correcting of $15 \mathrm{~nm}$ all the results obtained with the employed analysis.

$\dagger$ The precision in the detection of $r_{e}$ is enhanced by a parabolic fit of the extremum over 3 experimental points closer to the peak/valley. In this way a precision of 10 $\mathrm{nm}$ on $r_{e}$ is achieved.
}

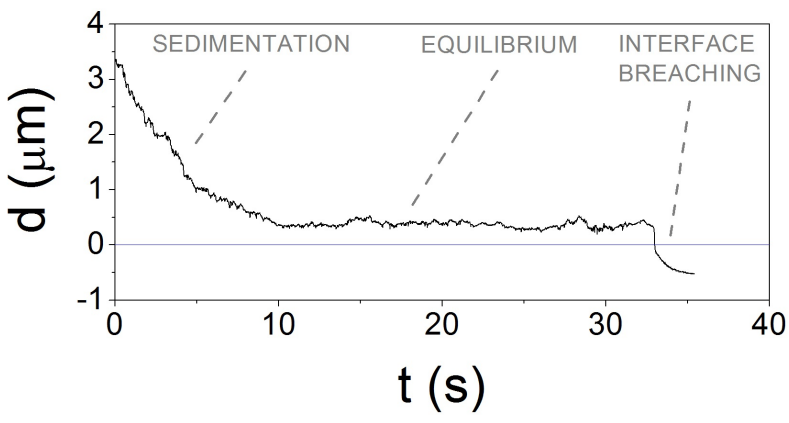

Fig. 5 Time evolution of the particle-interface distance $d$ for a spherical polystyrene particle with radius $4.5 \mu \mathrm{m}$ in a $5 \cdot 10^{-5} \mathrm{M}$ solution.

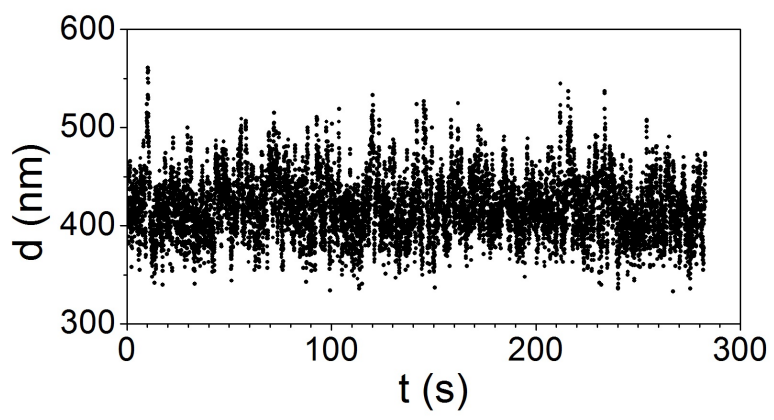

(a)

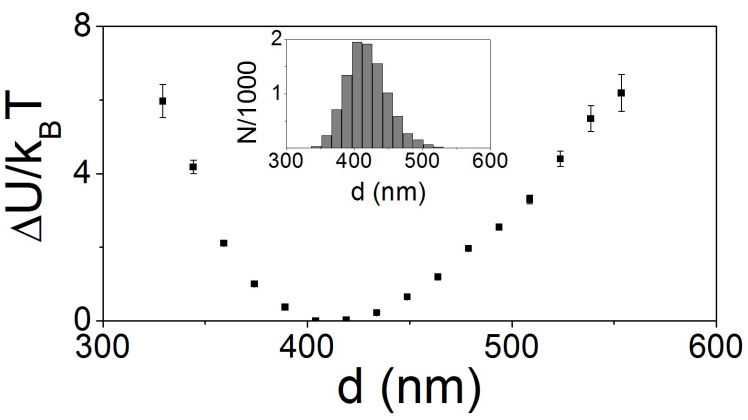

(b)

Fig. 6 (a) Temporal fluctuations of the gap distance $d(t)$ around its equilibrium value for a sulfate particle with radius $4.5 \mu \mathrm{m}$ in a $5 \cdot 10^{-5} \mathrm{M}$ solution. (b) Profile of the normalized potential energy difference $\Delta U(d) / k_{B} T$ obtained using eq. 3 and the histogram of the distances (in the inset) extracted from the trajectory in (a). The error bars in the normalized potential have been obtained propagating the incertitude on the probability distribution, which is due to the finite number of data and equal to $\sqrt{N(d)\left(1-\frac{N(d)}{N_{t o t}}\right)} / N_{t o t}$. 


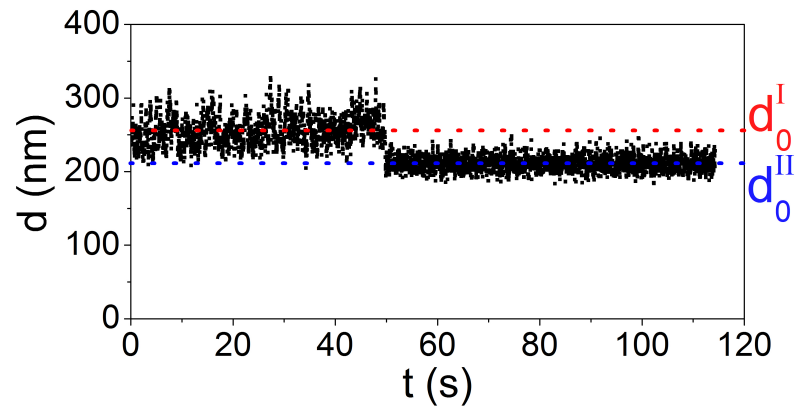

Fig. 7 Typical fluctuations around the first ( $d_{0}^{I}$, on the left) and the second ( $d_{0}^{I I}$, on the right) equilibrium position for a sulfate polystyrene particle of radius $4.5 \mu \mathrm{m}$ in a water solution of molarity $c=4.8 \cdot 10^{-4} \mathrm{M}$. The two equilibrium positions are at $d_{0}^{I}=250 \pm 5 \mathrm{~nm}$ and $d_{0}^{I I}=211 \pm 5 \mathrm{~nm}$. The transition from $d_{0}^{I}$ to $d_{0}^{I I}$ happens in about $300 \mathrm{~ms}$.

energy of the particle can be extracted using the Bolzmann equation (BE), 17 as reported in figure 6p. The BE relates the probability $p(d) \mathrm{d} d$ of finding the particle at a distance between $d$ and $d+\mathrm{d} d$ to the potential energy $U(d)$ of the particle. In the case of a large number of observations $\left(N_{t o t} \rightarrow \infty\right)$, the probability ratio $p(d) / p\left(d_{0}\right)$ can be approximated by the ratio $N(d) / N\left(d_{0}\right)$ between the number of observations in the bins centered in $d$ and in $d_{0}$ respectively. The BE can thus be written as:

$$
\frac{\Delta U(d)}{k_{B} T}=-\ln \frac{N(d)}{N\left(d_{0}\right)},
$$

where $\Delta U(d)=U(d)-U\left(d_{0}\right)$. Typical values of $N_{\text {tot }}$ in our experiments are about $10^{4}$, resulting in relative errors on $\Delta U(d)$ of the order of $1-10 \%$.

During the experimental campaign two kinds of equilibrium positions have been typically observed. The sedimenting particles stop at a first equilibrium distance $d_{0}^{I}$ of a few hundreds of nanometers and fluctuate around this location. With time, an increasing number of them is found at a distance $d_{0}^{I I}$ closer to the interface where they show much less fluctuations. This change is illustrated in figure 7 where we followed a particle initially located at a distance $d_{0}^{I}=250 \mathrm{~nm}$ from the interface which at a given time suddenly moves to the second equilibrium position $d_{0}^{I I}$ a few tens of nanometers closer to the interface.

Using BE (eq. 3) we extracted from the fluctuations the corresponding energy profiles $\Delta U^{I}$ and $\Delta U^{I I}$ (see figure 8) around the two equilibrium positions $d_{0}^{I}$ and $d_{0}^{I I}$. We find that the attractive branch in $\Delta U^{I I}$ (grey circles in fig 8 ) is steeper than the one of $\Delta U^{I}$ (black squares in fig 8), which in turn has the same slope of the gravitational potential expected to act on the particle (dashed lines). Moreover, the two potential wells overlap, meaning that for the same values of $d$ in the range $200 \mathrm{~nm}<d<250 \mathrm{~nm}$ there are two possible values of the potential energy, corresponding either to a repulsive or to an attractive force acting on the particle.

In general it is easier to find a particle in the second equilibrium position at high molarities and for carboxylated particles. The observation of more than 50 samples (about 5 measured par-

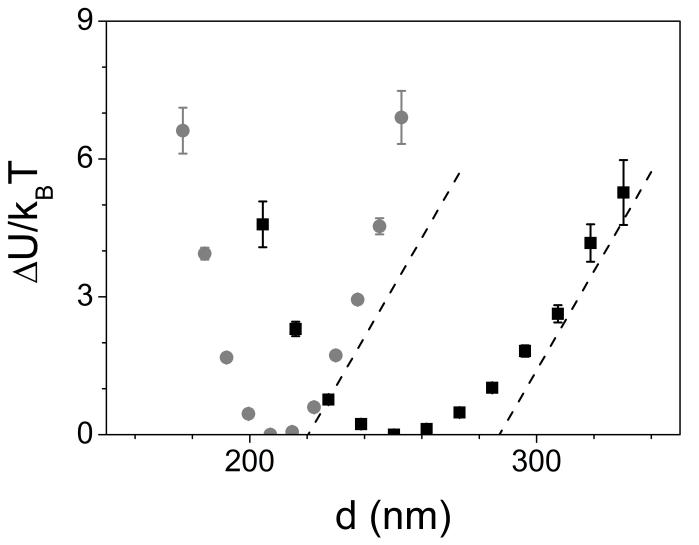

Fig. 8 Normalized potential energy difference $\Delta U / k_{B} T$ associated to the first (black squares) and to the second (grey circles) equilibrium positions obtained from $d(t)$ of figure 7 The dashed lines represent the expected gravitational potential. The error bars are obtained propagating the incertitude on the probability distribution due to the finite number of data.

ticles per sample) prepared at different molarities revealed that at $c<10^{-5} \mathrm{M}$ all sulfate particles, and most of the carboxylated ones, reside in the first equilibrium position for the whole duration of the experiments (few hours). On the other hand at larger molarity ( $c \geq 10^{-3} \mathrm{M}$ for sulfate and $c \geq 10^{-4}$ for carboxylated), after $100 \mathrm{~min}$, the totality of the observed particles populate the second equilibrium position. The number of particle adsorbed at the air-water interface follows the same trend: it is large for carboxylated particles at high molarity, while it becomes lower for sulfate particles.

The transition from the second equilibrium back to the first one is rarely observed during our measurements (3 events). The shape of the potential well $\Delta U^{I}$ in that case is the same before and after the stay in $d_{0}^{I I}$. Such observations indicate that the transition between the two equilibria is reversible and the energy $U^{I I}\left(d_{0}^{I I}\right)$ associated to the second minimum is lower than the one of the first $U^{I}\left(d_{0}^{I}\right)$.

In figure 9 the dependence of $d_{0}^{I}$ (black squares) and $d_{0}^{I I}$ (grey circles) on the molarity is reported for the sulfate particles. Each point is the averaged value of $d_{0}^{I}$ or $d_{0}^{I I}$ made over 5-20 particles. The equilibrium distance $d_{0}^{I}$ decreases with molarity: particles get closer to the interface when the ions concentration increases in the solution. For molarities larger than $10^{-4} \mathrm{M}$ (Debye screening length $\lambda_{D}$ lower than $30 \mathrm{~nm}$ ) the particle-interface distance is independent from molarity, suggesting that the electrostatic contribution to the particle-interface interaction is negligible at large ionic concentrations. The values of $d_{0}^{I I}$ follow the same trend as $d_{0}^{I}$, remaining systematically lower (of about $50 \mathrm{~nm}$ ) than $d_{0}^{I}$. At the lowest molarities the second equilibrium position is rarely observed.

\subsection{Analysis of the potential}

The measured particle-interface potential energies have been compared to a DLVO model which takes into account gravita- 


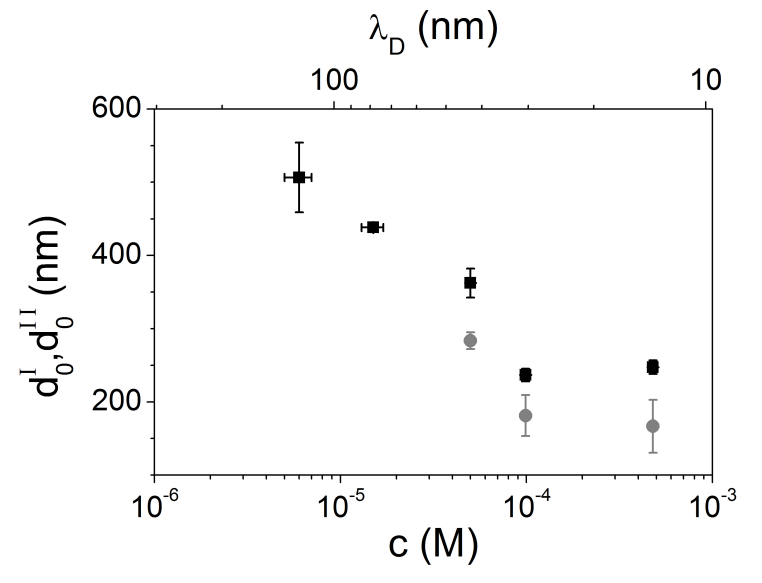

Fig. 9 Plot of $d_{0}^{I}$ (black squares) and $d_{0}^{I I}$ (grey circles) as a function of molarity for the sulfate particles. Each point is an average over the equilibrium positions of 5-20 particles measured in solutions at same molarity. Error bars on the vertical axis are the standard deviation of the averages, while the ones on the abscissa come from the experimental incertitudes in solutions preparation. Top scale reports the corresponding Debye screening length $\lambda_{D}=\sqrt{k_{B} T \varepsilon \varepsilon_{0} \cdot 10^{3} / 2 c N_{A} e^{2}}$, with $N_{A}$ the Avogadro number, $\varepsilon_{0}$ the vacuum permittivity and $\varepsilon$ the relative permittivity of water.

tional, van der Waals and double-layers interactions.

The particle is driven by its weight towards the interface in order to minimize the gravitational potential $U_{g}$ given by:

$$
U_{g}=m^{*} g d
$$

where $m^{*}$ is the effective mass of the particle: $m^{*}=4 \pi a^{3} \Delta \rho / 3$ with $\Delta \rho$ the density mismatch between the particle and the solution.

The van der Waals interaction is modelled using its exact expression for the plane-sphere geometry: 18

$$
U_{V d W}=\frac{A_{H}}{6}\left[\frac{a}{d}+\frac{a}{d+2 a}+\ln \left(\frac{d}{d+2 a}\right)\right],
$$

where the Hamaker constant $A_{H}$ is defined using the convention of positive values for repulsive van der Waals interactions. The value of $A_{H}$ in our system is experimentally determined considering the measured interaction in high molarity samples ( $c=4.8 \cdot 10^{-4} \mathrm{M}$ ), for which the electrostatics gives negligible contributions. For these samples we fitted the measured interaction energy close to $d_{0}^{I}$ with a potential energy given by $U_{g}+U_{V d W}$ (eq. 4 and 5), keeping $A_{H}$ and an additive constant as free parameter In figure 10 a typical example of the fit is reported. A very good agreement can be observed between the fitting function (line) and the experimental data (points). The mean value of the Hamaker constant found averaging over the fitted values of 7 dif-

$\$$ We only consider $d_{0}^{I}$ because is the one whose potential well can be successfully compared with $U_{g}+U_{V d W}$. As it will detailed below, $\Delta U^{I I}$ is incompatible with a potential having gravity as the only long-range attractive force.

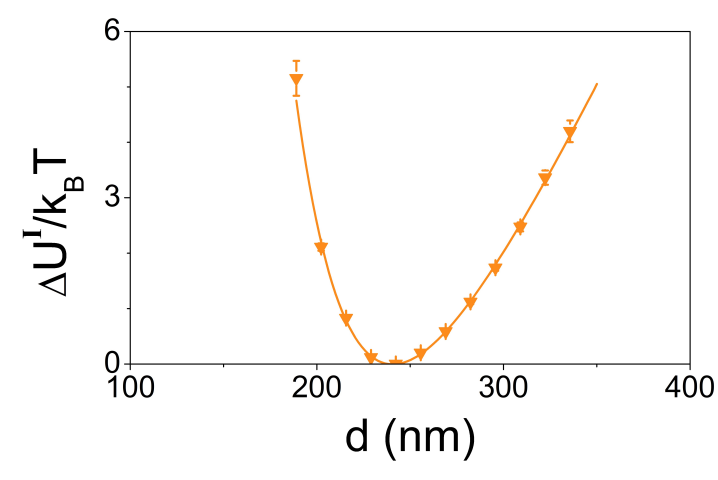

Fig. 10 Fit with the expression given by $U_{g}+U_{V d W}$ (line) of the normalized potential measured for a particle in $d_{0}^{I}$ in a solution at $c=4.8 \cdot 10^{-4} \mathrm{M}$ (points). The free fitting parameters are the Hamaker constant $A_{H}$ and an additive constant potential. The error bars are obtained propagating the incertitude on the probability distribution due to the finite number of data.

ferent particles is $A_{H}=2.0 \pm 0.4 \cdot 10^{-20} \mathrm{~J}$, comparable but slightly larger than the tabulated value of $1.57 \cdot 10^{-20} \mathrm{~J}$ for polystyrenewater-air systems. 19 The agreement is quite good considering that the surface groups present on the particle can change the effective Hamaker constant. 18 The determined $A_{H}$ can be considered independent from molarity at the relatively low ionic concentrations of our experiments. 20

A repulsive double-layers interaction is expected as both the air-liquid interface and the particles are negatively charged. For the choice of its expression we followed the arguments of Del Castillo et al. 4 and considered mixed electric boundary conditions, namely a fixed surface charge $\sigma_{q}$ for the particle and a fixed surface potential $\psi_{A L}$ for the air-liquid interface. Accordingly, the double-layers potential $U_{D L}^{\psi-\sigma}$ results in:21

$$
\begin{aligned}
U_{D L}^{\psi-\sigma} & =\pi a\left[\frac{2 \psi_{A L} \sigma_{q}}{\mathrm{k}}\left(\frac{\pi}{2}-\arctan \sinh (\mathrm{k} d)\right)-\right. \\
& \left.+\left(\frac{\sigma_{q}^{2}}{\mathrm{k}^{2} \varepsilon \varepsilon_{0}}-\psi_{A L}^{2} \varepsilon \varepsilon_{0}\right) \ln \left(1+e^{-2 \mathrm{k} d}\right)\right]
\end{aligned}
$$

where $\mathrm{k}=\lambda_{D}^{-1}$ is the reciprocal of the Debye screening length. Please note that eq. 6 is obtained in the Derjaguin and DebyeHückel approximations. 21 While Derjaguin limit $(\mathrm{k} a \gg 1$ and $d \ll a$ ) in our case is verified at all the considered molaritie $\$$ the Debye-Hückel condition for the linearization of the PoissonBoltzmann equation $\left(e \psi / k_{B} T \ll 1\right)$ is poorly satisfied 7 However, numerical calculations reported in literature ${ }^{22}$ showed that, in the limit of $\mathrm{k} a \gg 1$ and for $\mathrm{k} d>1$, the expression reported in eq. 6 overlaps with the exact numerical solution. As in our experiments $\mathrm{k} a>20$ and $\mathrm{k} d>5$, we can thus safely use relation 6 to fit the data.

$\S$ In the present system $\mathrm{k} a \geq 20$ and $d / a \leq 0.1$.

ๆ For sulfate and carboxylated particles we have that $e \psi / k_{B} T \sim 39$ and 8.6 respectively. 


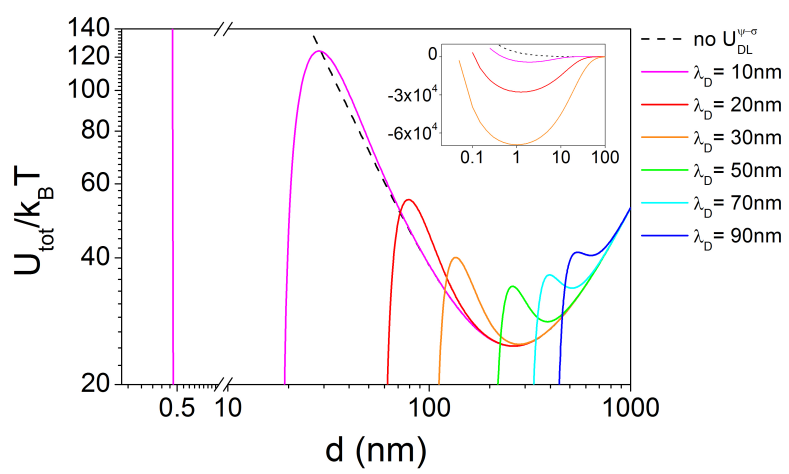

Fig. 11 Plot of $U_{\text {tot }}$ versus $d$ for a polystyrene sphere of radius $a=4.5 \mu \mathrm{m}$ and surface charge $\sigma_{q}=-5 \mathrm{mC} / \mathrm{m}^{2}$ interacting with an air-liquid interface at constant potential $\psi_{A L}=-1 \mathrm{mV}$ for an Hamaker constant $A_{H}=2 \cdot 10^{-20}$ $\mathrm{J}$. The potential $U_{\text {tot }}$ is reported in continuous lines for different values of $\lambda_{D}$. For comparison $U_{V d W}+U_{g}$ is also reported (black dashed line). In the inset, the potential $U_{V d W}+U_{g}$ and $U_{\text {tot }}$ for $\lambda_{D}=10,20$ and $30 \mathrm{~nm}$ are reported in linear scale to visualize the absolute minimum around $d=1$ $\mathrm{nm}$.

Considering all the contributions to the particle-interface interaction, the particle potential $U_{t o t}$ results in:

$$
U_{t o t}=U_{g}+U_{V d W}+U_{D L}^{\psi-\sigma}
$$

In order to discuss the role of the double-layers potential, in figure $11 U_{V d W}+U_{g}$ (dashed line) is compared to the total potential $U_{t o t}$ for different values of $\lambda_{D}$ in the experimental range of values (continuous lines). Because of van der Waals and doublelayers repulsions both potentials are strongly repulsive at very low $d$, while they are attractive at large $d$ due to gravity. Unlike $U_{V d W}+U_{g}$, however, $U_{\text {tot }}$ presents two minima. A first relative minimum at large $d$ (hundreds of nanometers), close to the one of $U_{V d W}+U_{g}$, and an absolute one (in the inset) at about $1 \mathrm{~nm}$ or less from the interface. An energy barrier from few $k_{B} T$ up to $100 k_{B} T$ (depending on molarity) separates the first minimum from the much deeper second one. The relative minimum originates from the balance of attractive gravity and repulsive van der Waals and double layers interactions. The short-range absolute minimum, on the other side, is a consequence of a fixed electrostatic potential at the air-water interface: in order to keep a constant potential, an inversion of interface electrical charges occurs at short particle-interface distances inducing a sign reversal of the double layers interaction. 421

Decreasing $\lambda_{D}$, the relative minimum of $U_{t o t}$ approaches the interface, until it reaches its lower value corresponding to the minimum of $U_{V d W}+U_{g}$. The dependence of the relative minimum position on $\lambda_{D}$ has the same trend as the experimental $d_{0}^{I}$ (fig. 9). At the same time, as $\lambda_{D}$ decreases, the energy barrier separating the minima increases and its position approaches to the interface. This model therefore predicts an easier crossing of the energy barrier at low molarities, in contrast with experimental observations. This hints to a different nature and origin of the experimental second minimum as electrostatics seems to prevent,

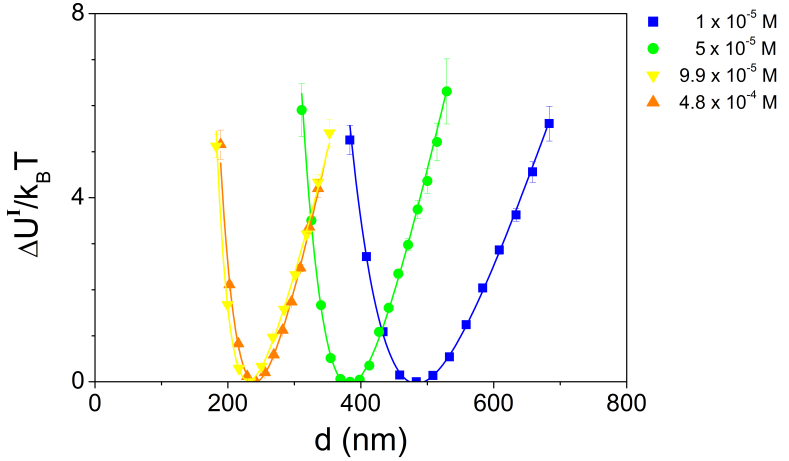

Fig. 12 Typical measured $\Delta U^{I}$ (points) with their best fits (lines) made with eq. 7 Data corresponds to four sulfate particles in solutions at different molarity: $4.8 \cdot 10^{-4} \mathrm{M}$ (orange triangles), $9.9 \cdot 10^{-5} \mathrm{M}$ (yellow triangles), $5 \cdot 10^{-5} \mathrm{M}$ (green circles) and $1 \cdot 10^{-5} \mathrm{M}$ (blue squares). The error bars are obtained propagating the incertitude on the probability distribution due to the finite number of data.

and not facilitate, its observation.

The measured $\Delta U^{I}$ has been fitted using eq. 7 with $a, \lambda_{D}, \psi_{A L}$ and an additive constant energy $U_{0}$ as fitting parameters. The density of the solution is known from tabulated data as a function of the molar concentration $c$ and the temperature $T .23 .24$ Figure 12 reports typical best-fit curves for four different sulfate particles in $\mathrm{NaCl}$ solutions at different molarities. For each data set (points), the corresponding fit (continuous line) is superimposed to the points. The agreement between model and data is good with a confidence higher than $95 \%$. Fits made with different boundary conditions for the double-layers potential, i.e. constant electric charge on both the particle and the interface, give similar results but with a lower confidence level.

The best fit values of $\psi_{A L}$ for the sulfate and carboxylated particles have an average value of $-1.3 \pm 0.2 \mathrm{mV}$ and $-1.1 \pm 0.2 \mathrm{mV}$ respectively. These averages are made over particles in solutions at concentrations lower than $10^{-4} \mathrm{M}$, for which the electrostatics carries significant contributions to $U_{t o t}$. The obtained air-liquid surface potentials are independent from the particle type as expected and in the range of values reported in literature, although smaller in modulus than most of them. ${ }^{3}$ Moreover, no significant dependence of $\psi_{A L}$ from the molarity has been observed (see figure 13), thus confirming the expected independence of the electric charge density at the air-liquid interface from the concentration of ions in the solution. 625

While the first experimental equilibrium $d_{0}^{I}$ is correctly accounted by the DLVO model, the second one experimentally found at hundreds of nanometers from the interface cannot be identified with the theoretical absolute minimum at $d<1 \mathrm{~nm}$ Previ-

|| For the sake of completeness, among the particles adsorbed at the interface, about $10 \%$ of them resided for few seconds at a tiny particle-interface distance (lower than experimental resolution of $10 \mathrm{~nm}$ ) before starting the interface breaching dynamics. This, and not the measured $d_{0}^{I I}$, could be compatible with the absolute minimum predicted by DLVO model. 


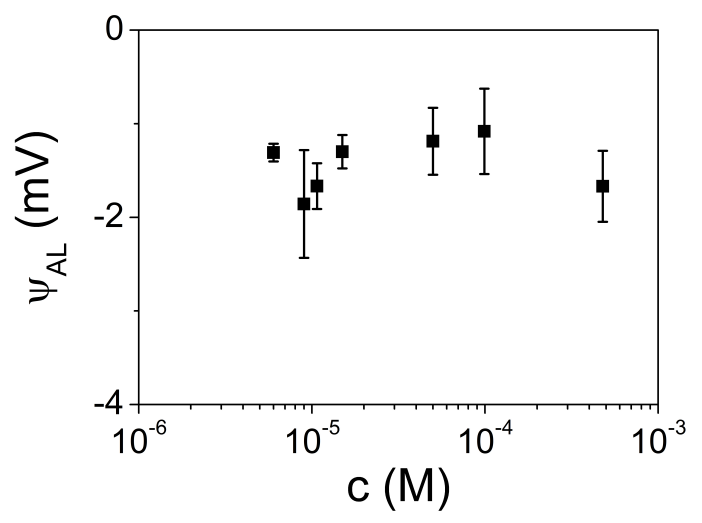

Fig. 13 Mean values of the air-water surface potential obtained from the fits as a function of the concentration $c$ of $\mathrm{NaCl}$ in the solution.

ous studies on the topic ${ }^{3 / 4} 7$ have never revealed the presence of such large distance second equilibrium position $d_{0}^{I I}$. Moreover, the measured coexistence of the two potentials for the same particleinterface distance demands to search for an interaction potential function of almost one more variable besides the particleinterface distance $d$. A first simple hint consists in an explicit dependence on time possibly originating from a contamination of the air-water interface changing the particle interaction. Such irreversible phenomenon is however not compatible with the observed reversible transition between the two potentials. In addition, surface tension control measurements made on the solutions in the same experimental conditions ensured that the surface remains clean for several hours after its preparation.

Looking for additional information on the system, we have considered the particle orientation with respect to the interface characterized by the zenithal out-of-plane (of the interface) angle $\theta_{s}$ and the azimuthal in-plane angle $\varphi_{s}$ (see figure 14). By tracking the position $\vec{r}_{c}$ of one crater with respect to the center of the interference pattern one can directly access to $\varphi_{s}$ and calculate $\theta_{s}$ as $\theta_{s}=\arcsin \left(r_{c} / a\right)$. It is important to remark that such craters are not related to the presence of the second equilibrium: carboxylated particles, devoid of such features, still show two equilibrium positions. Moreover, the radial position of trackable craters in the case of sulfate particles are not related to the particle equilibrium distance.

Figure 15 shows the zenithal orientation of a particle tracked during the sudden change of its position from $d_{0}^{I}$ (in red) to $d_{0}^{I I}$ (in blue). Because of thermal agitation, $\theta_{s}$ evolves in time. The square root of its Mean-squared Angular Displacement MSAD after $10 \mathrm{~s}$ is about $8^{\circ}$ when the particle fluctuates in the potential well $\Delta U^{I}$, while it drops down to $2^{\circ}$ (lower than the typical error bar of the measurement represented by the light blue area in figure 15) when it passes in the second minimum. Such a big difference is not accounted by hydrodynamics models which predict only a slight decrease of $4 \%$ of the MSAD when the particle goes from the first to the second equilibrium position ${ }^{28}$ due to the corresponding change in the particle-interface distance. On the other hand, azimuthal dynamics seems to follow hydrodynam-

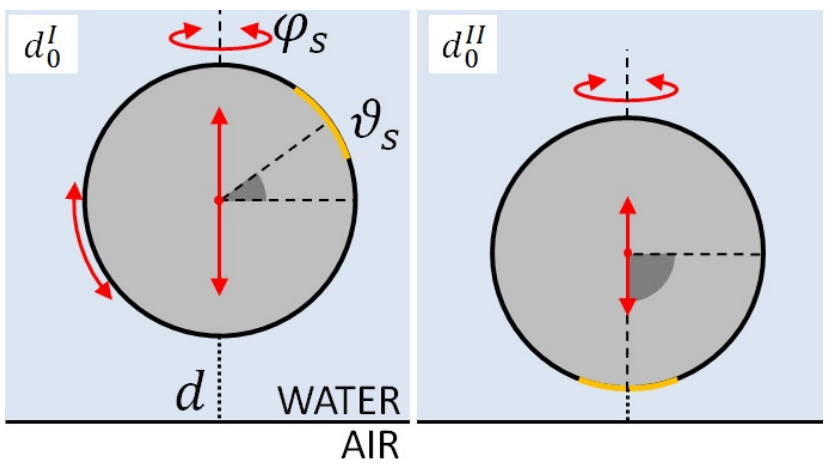

(a)

(b)

Fig. 14 Sketch of the possible physical origin of the second equilibrium position. $\theta_{s}$ is the zenithal angle corresponding to the out-of-plane (of the interface) rotation. $\varphi_{s}$ is the azimuthal angle of the in-plane (of the interface) rotation. The yellow arc represents the area of a surface heterogeneity. (a) First minimum: the heterogeneity is far from the interface and the particle can diffuse along all the three represented degrees of freedom $\left(d, \theta_{s}\right.$ and $\left.\varphi_{s}\right)$. (b) Second minimum: the heterogeneity is pointing towards the interface and the $\theta_{s}$ degree of freedom is frozen.

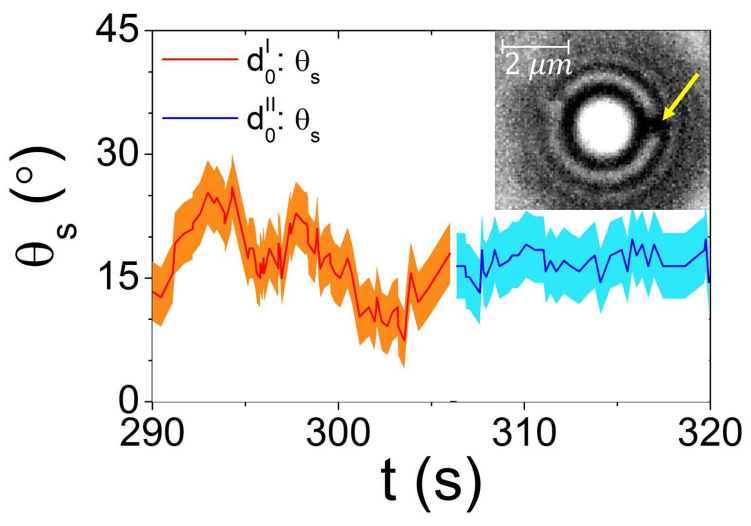

Fig. 15 Time evolution of the zenithal angle $\theta_{s}$. Red and blue lines correspond to $\theta_{s}$ in $d_{0}^{I}$ and $d_{0}^{I I}$ respectively. Orange and light blue areas represent the uncertainties in the measurement of $\theta_{s}$. In the inset the yellow arrow indicates on the contrast-enhanced interference pattern the feature tracked to recover particle orientation. 
ics predictions as during the same amount of time we measure a square root of the MSAD of $12^{\circ}$ and $11^{\circ}$ in the first and in the second equilibrium positions respectively. These results suggest that the zenithal orientation of the particle is free when the particle is in the first minimum while it is frozen in the second minimum (figure 14). The same behaviour has been observed for all the 45 sulfate particles presenting trackable defect features. The different angular dynamics in the two minima strongly suggests a potential energy depending on the particle orientation $\theta_{s}$. The potential $U\left(d, \theta_{s}\right)$ would therefore exhibit different profiles in $d$ for different values of $\theta_{s}$, possibly corresponding to the two observed equilibrium positions. Such angular dependence of the potential results in a torque on the particle and would emerge naturally if a spherical particle were not homogeneous all over its surface. Surface heterogeneities may eventually promote a larger than gravity particle-interface attraction as the one measured from the slope of the attractive branch of the interaction potential around the second minimum (see figure 8).

Considering the low experimental value of the ratio $d / a$ and the fast spatial decay of the DLVO interaction, only the side of the particle turned towards the air-liquid interface is expected to be relevant for the particle-interface interaction. A local surface heterogeneity having an enhanced attraction to the interface is therefore expected to impose an angular lock of the particle in the second equilibrium position. Surface heterogeneities number and dimensions can be estimated from the statistical analysis of the first to second minimum transitions and from the fluctuations amplitude around the second minimum. From the angular fluctuation amplitudes and the particles radius one obtains heterogeneities sizes of the order of $10-100 \mathrm{~nm}$. On the other hand, to reach the second equilibrium position a particle in the first one has to rotationally diffuse in a way to expose one heterogeneity to the interface. From the known value of the particle rotational diffusion coefficient, 2829 and the observation that after $30 \mathrm{~min}$ utes almost all the particles are found in the second minimum, one can estimate that just one heterogeneity per particle is sufficient to explain the first to the second minimum transition (more details are given in the Supplementary Informations).

Let us discuss such findings in the framework of the model considered in eq. 77. The increase of the slope of the attractive branch of $\Delta U^{I I}$ well beyond the gravity value (figure 8) rules out any possible explanation of a rotational lock related to a gravitational torque due to any morphological asphericity of the particle. Furthermore, the observation that the second minimum appears more often in solutions at high $\mathrm{NaCl}$ molarities and with the less charged carboxylated particles, when the electrostatics is weaker, supports a van der Waals origin of the effect. This will be the case if air nanobubbles are pinned on the surface of the particle. Previous AFM studies of flat polystyrene surfaces have revealed the presence of flattened air nanobubbles strongly pinned on the substrate. $\frac{30 \mid 31}{30}$ Rotations bringing a region of particle's surface rich in air-bubbles close to the air-water interface can induce an additional attraction since the two gaseous regions attract by van der Waals interaction. Preliminary results reported in the Supplementary Informations show indeed an easier adsorption at the interface, correlated to an easier transition from the first to the second equilibrium, for particles in degassed samples.

Note that this interpretation is compatible with previous experimental results using different interfaces. For solid-water 32 and oil-water ${ }^{8}$ interfaces, indeed, the presence of air nanobubbles would induce a repulsive (and not attractive) additional van der Waals interaction, preventing the formation of a second minimum. Moreover, the verified $\theta_{s}$ dependency of the potential makes much more difficult the observation of $d_{0}^{I I}$ in AFM measurements, where the probing particle is stuck on the cantilever.

A simple van der Waals model considering a nanometer-thick air layer on the surface of a planar perfectly flat polystyrene wall and neglecting retardation effects actually shows a decrease of the equilibrium distance ${ }^{33}$ but fails, however, in describing the observed potential. A deeper analysis of the effect of air-bubbles on the particle-interface interaction should therefore overcome simple approximations and consider either retardation effect 34 and surface roughness both in the context of van der Waals interaction through multilayered media. $\frac{35}{35}$

At the same time, a further test of the role of nanobubbles would be given by the experimental study of microparticles engineered to promote pinning of air bubbles. Such condition can be achieved through a physical modification of the particles surface by making them superhydrophobic, by changing their topography with the adsorption of positively charged nanoparticles or by a chemical treatment in order to form asperities through erosion at nanometric scale. 36 Another possible way could be a chemical treatment of microparticles to enhance the nanobubbles formation, for example evaporating hydrophobic silanes on negatively charged silica particles. $\frac{39}{39}$

\section{Conclusions}

In this work we have measured the interactions between a spherical microparticle and an air-water interface. The particle tracking close to the interface, made with a specifically designed Dual Wave Reflecting Interference Microscopy setup, allows to measure the particle-interface interaction. Surprisingly, in most cases two equilibrium positions are observed. These distinct equilibria are qualitatively the same for each measured particle thus appearing a quite general feature of such physical system. The measured interaction potential associated to the farthest position from the interface is well reproduced by a classic DLVO model completed with an attractive gravitational energy. In this position the particle is found to freely rotate as expected. In contrast, around the equilibrium closest to the interface the attractive branch of the potential cannot be accounted for gravity alone and the particle orientation is frozen. The system behaviour as a function of the solution molarity indicates the decisive role played by van der Waals interaction in the appearance of the closest to the interface equilibrium position. We suggested that this novel behaviour may be related to heterogeneities of the particle surface. Among possible origins, pinned air nanobubbles may provide both the further van der Waals attraction needed and the independence from the surface details of each single particle. The presence of the closest minimum and the particle interface breaching both appear more easily in high molarity solutions and for less charged particles, suggesting a possible connection between them via the discussed 
extra van der Waals attraction. Such an attraction could therefore facilitate the particle interface breaching in these systems. This remarks appears to be of paramount interest in the studies of interface breaching since the classic DLVO model would always prevent negatively charged microparticles from being adsorbed at the air-water interface. We believe that such experimental findings will further stimulate a deeper theoretical analysis of the interaction between particles with heterogeneous surfaces and the air-water interface.

\section{Conflicts of interest}

There are no conflicts to declare.

\section{Acknowledgements}

We gratefully acknowledge the financial support of ANR Surfanicol (ANR14-CE07-0039-01) and Labex Numev (n ${ }^{\circ}$ 2014-2-44). We also thank Michel Ramonda for the AFM measurements and Martin In and Domenico Truzzolillo for fruitful discussions.

\section{Notes and references}

1 C. C. Berton-Carabin and K. Schroën, Annual review of food science and technology, 2015, 6, 263-297.

2 Y. Xing, X. Gui, L. Pan, B.-E. Pinchasik, Y. Cao, J. Liu, M. Kappl and H.-J. Butt, Advances in Colloid and Interface Science, 2017, 246, 105-132.

3 R. A. Pushkarova and R. G. Horn, Langmuir, 2008, 24, 87268734.

4 L. A. Del Castillo, S. Ohnishi, S. L. Carnie and R. G. Horn, Langmuir, 2016, 32, 7671-7682.

$5 \mathrm{~J} . \mathrm{K}$. Beattie, in The Intrinsic Charge at the Hydrophobe/Water Interface, 2011, vol. 2, pp. 153 - 164.

6 K. Marinova, R. Alargova, N. Denkov, O. Velev, D. Petsev, I. Ivanov and R. Borwankar, Langmuir, 1996, 12, 2045-2051.

7 E. Taran, M. A. Hampton, A. V. Nguyen and P. Attard, Langmuir, 2009, 25, 2797-2803.

8 L. Helden, K. Dietrich and C. Bechinger, Langmuir, 2016, 32, 13752-13758.

9 R. J. Oetama and J. Y. Walz, Journal of colloid and interface science, 2005, 284, 323-331.

10 J. Schilling, K. Sengupta, S. Goennenwein, A. R. Bausch and E. Sackmann, Physical Review E, 2004, 69, 021901.

11 L. Limozin and K. Sengupta, ChemPhysChem, 2009, 10, 27522768.

12 G. Wiegand, K. R. Neumaier and E. Sackmann, Applied optics, 1998, 37, 6892-6905.

13 D. M. Kaz, R. McGorty, M. Mani, M. P. Brenner and V. N. Manoharan, Nature materials, 2012, 11, 138.

14 A. Ibrahim, H. Ohshima, S. A. Allison and H. Cottet, Journal of Chromatography A, 2012, 1247, 154-164.

15 J. Eastoe and J. Dalton, Advances in colloid and interface science, 2000, 85, 103-144.
16 N. Matubayasi, H. Matsuo, K. Yamamoto, S.-i. Yamaguchi and A. Matuzawa, Journal of colloid and interface science, 1999, 209, 398-402.

17 B. M. Alexander and D. C. Prieve, Langmuir, 1987, 3, 788795.

18 J. N. Israelachvili, Intermolecular and surface forces, Academic Press London; San Diego, 2nd edn, 1991.

19 D. C. Prieve and W. B. Russel, Journal of Colloid and Interface Science, 1988, 125, 1-13.

20 J. Marra, Journal of colloid and interface science, 1986, 109, 11-20.

21 G. Kar, S. Chander and T. Mika, Journal of Colloid and Interface Science, 1973, 44, 347-355.

22 P. Warszyński and Z. Adamczyk, Journal of colloid and interface science, 1997, 187, 283-295.

23 EngineeringToolBox, Water - Density, Specific Weight and Thermal Expansion Coefficient, 2003 (accessed December 3, 2017).

24 EngineeringToolBox, Density of aqueous solutions of inorganic sodium salts, 2017 (accessed December 3, 2017).

25 M. Takahashi, The Journal of Physical Chemistry B, 2005, 109, 21858-21864.

26 R. Zimmermann, S. Dukhin and C. Werner, The Journal of Physical Chemistry B, 2001, 105, 8544-8549.

27 C. Stubenrauch and R. Von Klitzing, Journal of Physics: condensed matter, 2003, 15, R1197.

28 K. Danov, T. Gurkov, H. Raszillier and F. Durst, Chemical Engineering Science, 1998, 53, 3413-3434.

29 L. D. Landau and E. M. Lifshitz, Volume 6 of Course of Theoretical Physics: Fluid Mechanics, Pergamon Press, 1987.

30 D. Li and X. Zhao, Colloids and Surfaces A: Physicochemical and Engineering Aspects, 2014, 459, 128-135.

31 D. Lohse, X. Zhang et al., Reviews of modern physics, 2015, 87, 981.

32 S. G. Flicker, J. L. Tipa and S. G. Bike, Journal of colloid and interface science, 1993, 158, 317-325.

33 N. Mishchuk, J. Ralston and D. Fornasiero, The Journal of Physical Chemistry A, 2002, 106, 689-696.

34 J. Gregory, Journal of Colloid and Interface Science, 1981, 83, 138-145.

35 B. Ninham and V. Parsegian, The Journal of Chemical Physics, 1970, 52, 4578-4587.

36 M. Zanini, C. Marschelke, S. E. Anachkov, E. Marini, A. Synytska and L. Isa, Nature communications, 2017, 8, 15701.

37 N. Hassan, A. Stocco and A. Abou-Hassan, The Journal of Physical Chemistry C, 2015, 119, 10758-10765.

38 A. San-Miguel and S. H. Behrens, Langmuir, 2012, 28, 12038-12043.

39 S. Karpitschka, E. Dietrich, J. R. Seddon, H. J. Zandvliet, D. Lohse and H. Riegler, Physical review letters, 2012, 109, 066102. 